\title{
Impact of chosen force fields and applied load on thin film lubrication
}

\author{
Thi D. TA ${ }^{1, *}$, Hien D. TA ${ }^{2}$, Kiet A. TIEU ${ }^{1}$, Bach H. TRAN ${ }^{1}$ \\ ${ }^{1}$ School of Mechanical, Materials, Mechatronics, and Biomedical Engineering, University of Wollongong, NSW 2522, Australia \\ ${ }^{2}$ Ho Chi Minh City University of Technology and Education, Ho Chi Minh City 800010, Vietnam \\ Received: 06 July 2020 / Revised: 01 September 2020 / Accepted: 14 October 2020 \\ (C) The author(s) 2020 .
}

\begin{abstract}
The rapid development of molecular dynamics (MD) simulations, as well as classical and reactive atomic potentials, has enabled tribologists to gain new insights into lubrication performance at the fundamental level. However, the impact of adopted potentials on the rheological properties and tribological performance of hydrocarbons has not been researched adequately. This extensive study analyzed the effects of surface structure, applied load, and force field (FF) on the thin film lubrication of hexadecane. The lubricant film became more solid-like as the applied load increased. In particular, with increasing applied load, there was an increase in the velocity slip, shear viscosity, and friction. The degree of ordering structure also changed with the applied load but rather insignificantly. It was also significantly dependent on the surface structure. The chosen FFs significantly influenced the lubrication performance, rheological properties, and molecular structure. The adaptive intermolecular reactive empirical bond order (AIREBO) potential resulted in more significant liquid-like behaviors, and the smallest velocity slip, degree of ordering structure, and shear stress were compared using the optimized potential for liquid simulations of united atoms (OPLS-UAs), condensed-phase optimized molecular potential for atomic simulation studies (COMPASS), and ReaxFF. Generally, classical potentials, such as OPLS-UA and COMPASS, exhibit more solid-like behavior than reactive potentials do. Furthermore, owing to the solid-like behavior, the lubricant temperatures obtained from OPLS-UA and COMPASS were much lower than those obtained from AIREBO and ReaxFF. The increase in shear stress, as well as the decrease in velocity slip with an increase in the surface potential parameter $\zeta$, remained conserved for all chosen FFs, thus indicating that the proposed surface potential parameter $\zeta$ for the COMPASS FF can be verified for a wide range of atomic models.
\end{abstract}

Keywords: molecular dynamics simulation; force fields; applied load; tribofilm; hydrocarbon

\section{Introduction}

Hexadecane lubricant, which is widely used in many tribological systems, has been analyzed in several theoretical studies over the last two decades, particularly in studies on steel surfaces [1-10]. Under confined conditions, thin hexadecane film behaves differently from its bulk form. Theoretical investigations revealed the formation of layering and slip phenomena at solid-liquid interfaces [2, 6-8], which depend on many variables such as surface roughness $[2,6,7]$, lubricant additives [4], surface structure and film thickness [2], as well as applied pressure [10]. Moreover, an increase in the solid-liquid adhesive strength significantly reduced the slip phenomenon at the interface $[4,11,12]$, whereas the slip occurred

* Corresponding author: Thi D. TA, E-mail: thi@uow.edu.au 
internally between the molecular layers within the lubricant [11]. Zheng et al. [10] determined the superior anti-wear performance of the long alkane chains and concluded that they behaved more solid-like than other shorter alkanes.

Although iron oxides are commonly found on mild steel strip surfaces during cold rolling, studies on the lubrication performance of hexadecane on steel surfaces at the atomic level remain inconclusive. Although several studies were conducted on the confined shear of hydrocarbons between metal surfaces, only a few of them considered the metal oxide surfaces in molecular dynamics (MD) simulations $[1,2,4]$. To characterize the adhesive strength between alkane and metal/metal oxide surfaces, Manias et al. [13] and Steven et al. [14] proposed a factor $k$, which varies in a wide range, to present the different interaction strengths between the alkane and the confined surfaces. Berro et al. [15] and Savio et al. [1, 2] adopted this factor, which is based on the energy difference per $\mathrm{CH}_{2}$ group obtained from experimental measurements of alkanes adsorbed on $\mathrm{Ni}$ [16], Ag [17], and Au [18] surfaces, to compare the tribological behavior of alkanes between different metal $(\mathrm{Au}, \mathrm{Fe})$ and metal oxide $\left(\mathrm{CuO}, \mathrm{Fe}_{2} \mathrm{O}_{3}\right)$ surfaces. Ta et al. [19] presented a more practical adhesive strength between hydrocarbons with iron and iron oxide surfaces by deriving an interfacial force field (FF) from ab initio calculations.

In MD simulations, different conventional and reactive potentials have been applied to hydrocarbons. However, studies are yet to be carried out to ascertain their influence on tribological performance and degree of layering order. In a few notable attempts, the non-equilibrium MD simulations indicated that the condensed-phase optimized molecular potential for atomic simulation studies (COMPASS [20]) FF predicts a higher zero-shear viscosity of hexadecane than assisted model building with energy refinement (AMBER) 96 [21, 22]. A benchmark was also set in terms of density and viscosity by Ewen et al. [23], who showed that united-atom (UA) FFs can accurately reproduce experimental density, whereas viscosity was significantly underpredicted compared to the all-atom (AA) model. They explained that the significant underestimation of viscosity by UA FFs suggested that the molecules moved past one another more easily, which indicates that the coefficient of friction ( $\mathrm{CoF}$ ) calculated for confined systems using UA FF will also be under-predicted. Although classical FFs can accurately model the physical processes of molecular systems under certain conditions, their potential functions are unable to reproduce the chemical reactions of these systems. Additionally, these FFs are generally poorly transferable when the properties of interest were not directly parameterized.

In this study, we considered the effects of the applied load and interfacial crystalline structure of iron compounds on the molecular structure and lubricability of hydrocarbon tribofilms. This study also aims to analyze the effect of available conventional and reactive potentials in predicting the tribological properties of hexadecane. The resulting article is organized in the following order. First, Section 2 presents the implemented methodology and relevant parameters for MD modeling. After that, Section 3 discusses the influence of loading pressure and crystalline structure on the rheological properties of hexadecane under confined shear conditions, as well as the impact of chosen FFs on the ordering structure and tribological properties of hydrocarbons. Finally, a conclusion is presented in Section 4.

\section{Methodology}

The MD simulation was performed using an AA model, in which the methyl groups of $\mathrm{CH}_{2}$ and $\mathrm{CH}_{3}$ were presented with the explicit atoms. COMPASS FF was applied to the lubricant [20], whereas Buckingham FFs were applied to the $\mathrm{FeO}$ and $\mathrm{Fe}_{2} \mathrm{O}_{3}$ surfaces. The embedded-atom method/Finnis-Sinclair potential (EAM/FS) was applied to the Fe (100) surface [24]. The confined shear process, as illustrated in Fig. 1, was implemented using the large-scale atomic/ molecular massively parallel simulator (LAMMPS) code [25], while the initial configuration was constructed in Material Studio 7.0 (MS 7.0). The parameters for the Buckingham potential, as reported by Guillot et al., are presented in Table S1 in the Electronic Supplementary Material (ESM) $[26,27]$. The interactions between surface atoms with fluid atoms, as well as between different lubricant 


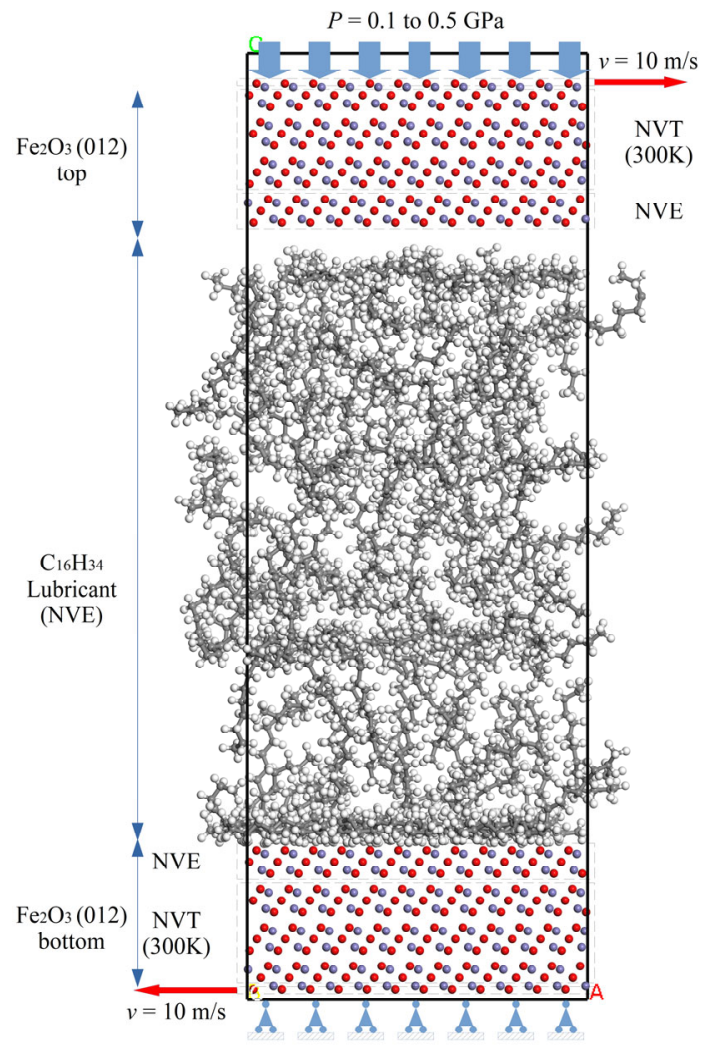

Fig. 1 Typical confined-shear model with hexadecane lubricant allocated between $\mathrm{Fe}$ (100), $\mathrm{FeO}$ (100), $\mathrm{Fe}_{2} \mathrm{O}_{3}$ (001), and $\mathrm{Fe}_{2} \mathrm{O}_{3}$ (012) surfaces with applied normal load and sliding velocity in the top surface while the bottom surface was constrained in the $z$-direction. This model was constructed in MS 7.0. NVT: Canonical ensemble with constant system's number of particles $(\mathrm{N})$, volume $(\mathrm{V})$, and temperature $(\mathrm{T})$; NVE: Microcanonical ensemble with constant system's number of particles $(\mathrm{N})$, volume $(\mathrm{V})$, and energy $(\mathrm{E})$

molecules, were determined using a Lenard-Jones 9-6 (L-J 9-6) potential with a cut-off distance of 12.5 Å combined with a long- range Columbic interaction. This long-range Columbic interaction adopted the particle-particle particle-mesh (PPPM) method with a desired relative error of $10^{-4}$ in forces, and the slab technique with a ratio of 3 , which is the ratio between the total extended volume used in the $2 \mathrm{D}$ approximation and the volume of the simulation domain [28]. The combination rules for this L-J 9-6 potential were applied to the interaction between the lubricant atoms, as well as between the lubricant and surface, which are expressed as Eqs. (S1) and (S2) in the ESM. To investigate the role of the applied load, COMPASS FF was used to describe the atomic interactions between hexadecane molecules. Theoretical MD simulations indicated that this FF applied to several substances and was commonly used to investigate the adsorption energy between organic molecules on metal oxide surfaces [29-32]. The functional forms and expressions for this FF are described in Table S2 in the ESM while the corresponding parameter values can be found in Ref. [20].

Interfacial interaction is a critical factor that affects the structure of organic molecules on the surface, as well as its rheological properties under confined conditions. Based on the adsorption energy between ethane and the iron surface, measured by Govender et al., the interaction between alkanes and iron was derived from $a b$ initio calculations, as shown in Fig. S1 in the ESM [33]. The procedure for calibrating this interaction parameter was implemented using their (Govender et al. [33]) work with L-J 9-6 potential. This parameterization was carried out at an absolute zero temperature. The ethane molecule and two upper iron layers were relaxed, whereas the rest were fixed. To obtain adsorption energy values consistent with Govender's result, the well-depth energy of iron $\left(\varepsilon_{\mathrm{Fe}}\right)$ for L-J 9-6 interactions between the surface and lubricant was maintained in the range of $0.02-0.05 \mathrm{eV}$. The dependence of the adhesive strength between the ethane and iron surface on the well-depth energy $\varepsilon_{\mathrm{Fe}}$ is presented in Fig. S2 in the ESM, which shows that the $\varepsilon_{\mathrm{Fe}}$ value of $0.0425 \mathrm{eV}$ appropriately represents the adsorption energy, $E_{\text {ad }}=-0.23 \mathrm{eV}$, obtained from previous $a b$ initio calculations. The non-bond interaction parameters between the iron oxide surfaces and hexadecane (Table S3 in the ESM) were obtained from the COMPASS FF and semi-ionic oxide model proposed by Zhao et al. [34].

As shown in Fig. 1, the molecular model was constructed with hexadecane allocated between the $\mathrm{FeO}(100)$ surfaces with a system domain of approximately $35 \AA \times 35 \AA \times 60 \AA$. Periodic boundary conditions were also applied in the lateral directions. To avoid self-interaction with its periodic images, the simulation domain was assigned a width twice the molecular chain length of hexadecane. This chosen system domain was similar to those presented by 
Berro et al. [35] and Jabbarzadeh et al. [36]. Each surface, cleaved from its regular crystalline lattice, had a thickness of approximately $10 \AA$, whereas the initial thickness of the lubricant was $40 \AA$. Although the $\mathrm{Fe}_{2} \mathrm{O}_{3}$ (001) surface has three chemically distinct terminations, in this study, it was set up by a single iron termination because this termination had the most stable surface configuration [37-39]. Details of the domain sizes and number of hexadecane molecules for each confined shear model are presented in Table 54 in the ESM.

The established model underwent tribological conditions, in which an applied pressure was introduced to the upper surface. To monitor its influence on the rheological and structural properties of hexadecane, this applied pressure varied in the range of 100$500 \mathrm{MPa}$. This MD simulation includes three main stages. The model was relaxed for $0.5 \mathrm{~ns}$ to neglect poor atom contacts during the first relaxation stage. Subsequently, a dynamic stage was introduced in the next $0.5 \mathrm{~ns}$ with an applied pressure on the top region to compress the model, while a fixed constraint was applied at the bottom region. To continuously conduct the frictional heat generated owing to compression and achieve an equilibrium condition, the temperature of the system was kept thermostatic at $300 \mathrm{~K}$ at the thermostatic layers on the surfaces. The NVT ensemble with a damping coefficient of 100 fs was used for this ensemble. In contrast, the NVE ensemble was applied to lubricant, as it was subjected to a constantly applied pressure. Finally, in the confined process, the surfaces were moved in opposite directions along the $x$-direction at a speed of $10 \mathrm{~m} / \mathrm{s}$ for $10 \mathrm{~ns}$, whereas the constraints of loading pressure, NVT ensemble for the surfaces, and NVE ensemble for the lubricant were retained. A time step of $1.0 \mathrm{fs}$ was applied to this MD simulation.

To analyze the influence of the selected FFs, different potentials, from the simplest UA model to an advanced reactive FF, were used. The UA potential employed in this work was the optimized potential for liquid simulations of united atoms (OPLS-UA) derived by Jorgensen and Tirado-Rives. [40], whereas COMPASS [20], adaptive intermolecular reactive empirical bond order (AIREBO) [41], and ReaxFF [42] were used for the AA models. Note that the applied FFs for the surfaces and their interactions with hydrocarbons were similar in all cases to be solely considered for the role of the lubricant model [22]. A comparison between these FFs was carried out based on the analysis of molecular structure, velocity gradient, shear stress, and slip ratio.

\section{Results}

\subsection{Influences of the applied load}

When the tribosystem was subjected to shear forces and applied pressure, the lubricant density increased gradually with the simulation time. Figure S3 in the ESM shows the time evolution of the atomic mass density, which oscillates during the first 100 ps owing to the instant impact of the applied load and becomes more stable after $11 \mathrm{~ns}$. This figure also reveals that although an increase in applied pressure induces a subsequent increase in oscillation amplitude at the beginning of the loading process, the oscillation amplitude becomes more stable during the sliding process.

The average lubricant density in Fig. 2(a) indicates a linear correlation between the lubricant density and the applied load, as a higher pressure induces an increase in the density of confined hexadecane. This observation agrees with previous investigations by Martini et al. $[8,10]$. The reduction in film thickness due to the applied pressure (Fig. 2(b)) could be the reason for the density increase in Fig. 2(a). However, a slight difference appeared among the averaged lubricant densities on different surfaces. This discrepancy may be due, in part, to the difference in the domain size of each surface using the same dimensions, number of lubricant molecules, and applied load for this comparative analysis. Additionally, the difference in the lattice constant for each surface material resulted in an apparent difference in surface dimensions (Table S4 in the ESM). However, the main source for this discrepancy is the difference in the ordering structure depending on the surface type.

Under relative sliding, kinetic energy was transferred into the fluid via the adhesive forces between the surface and lubricant $[43,44]$. This resulted in the formation of layers or ordering behavior of molecules adjacent to the surfaces. Figure 3 indicates that 

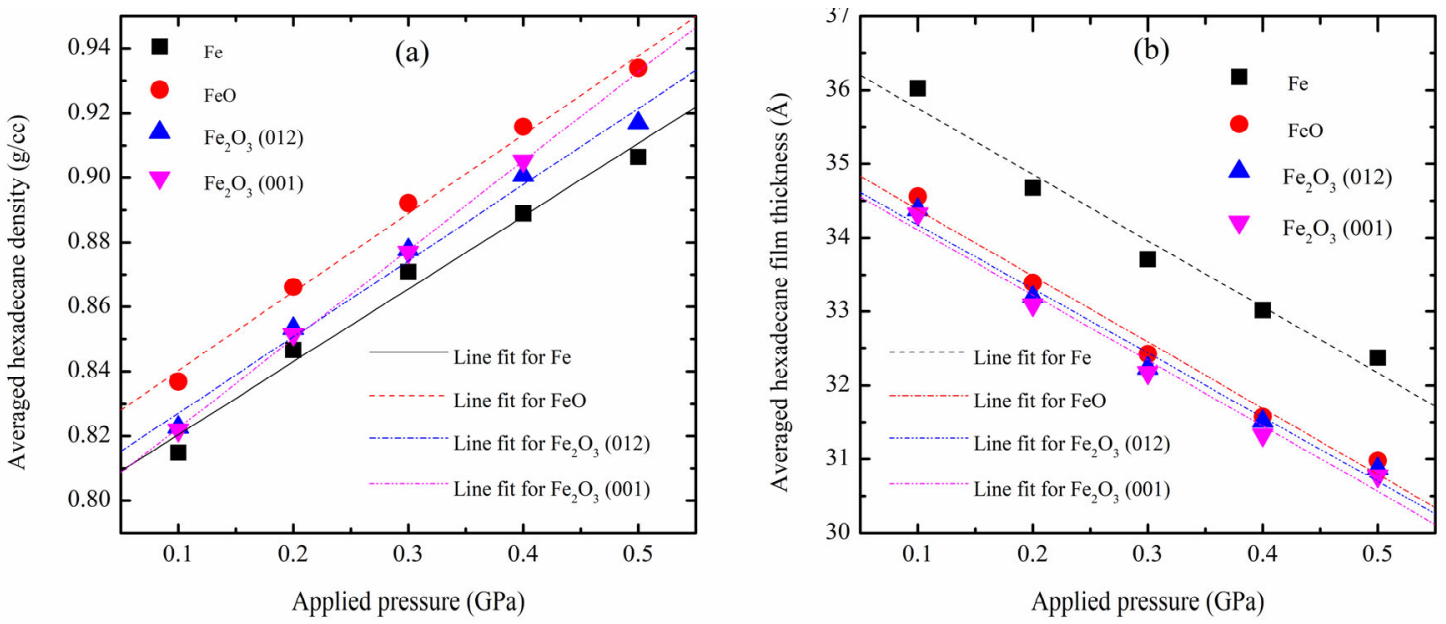

Fig. 2 Dependence of (a) time-averaged hexadecane film density and (b) film thickness on applied load for different iron compounds.
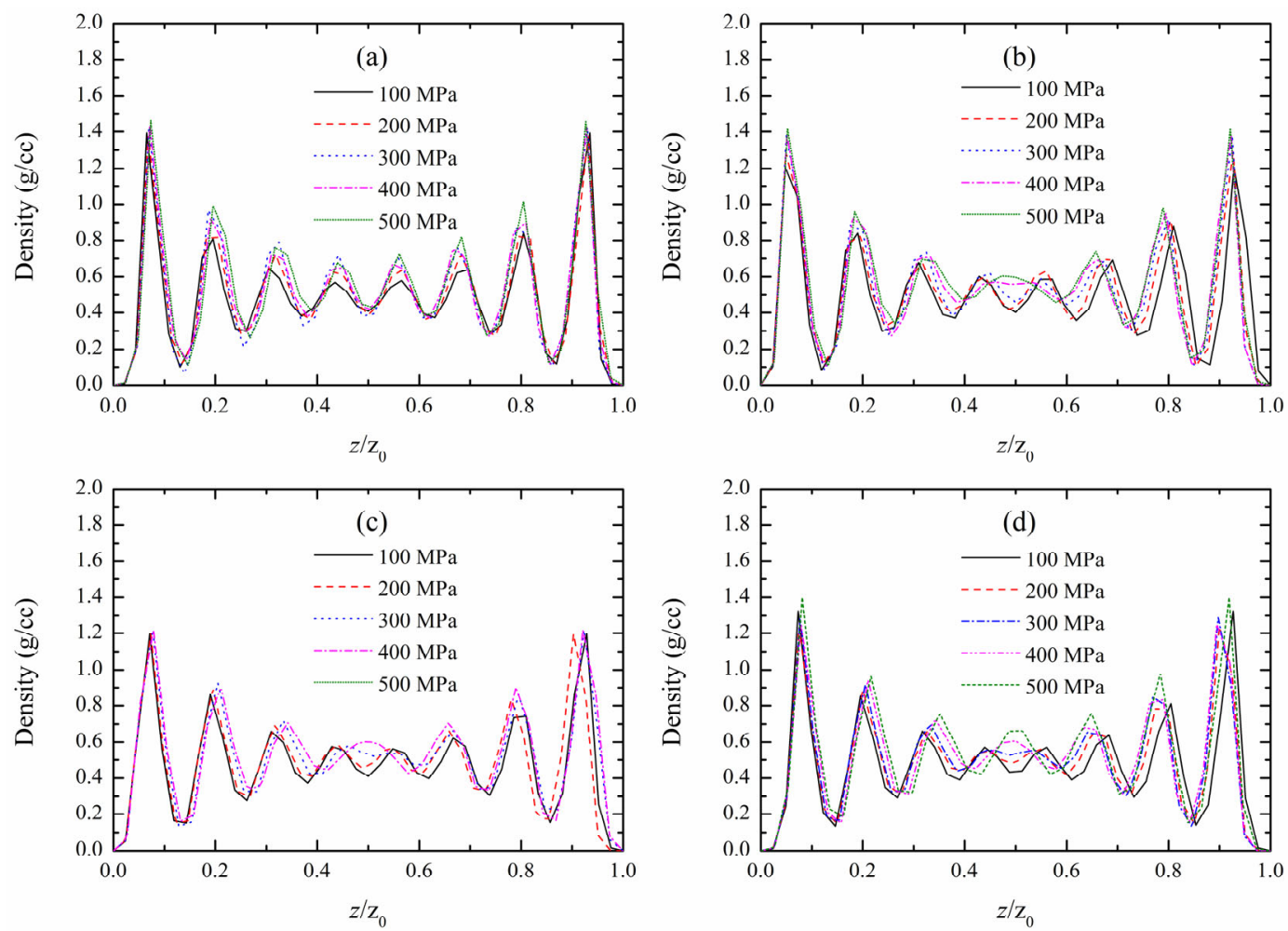

Fig. 3 Density profile across film thicknesses in the case of (a) $\mathrm{Fe}(100)$, (b) $\mathrm{FeO}(100)$, (c) $\mathrm{Fe}_{2} \mathrm{O}_{3}(001)$, and (d) $\mathrm{Fe}_{2} \mathrm{O}_{3}(012)$ surfaces at different applied loads. $z$ is the relative coordinate in $z$-direction from the bottom surface and $z_{0}$ is the lubricant film thickness.

although this layering density profile oscillates with the largest amplitude at the solid-fluid interfaces, its intensity gradually attenuates toward the middle regime of the lubricant film. This observation is consistent with Refs. [1, 2, 7, 10, 36, 45, 46]. The thickness of the film decreases with increasing applied pressure, which results in a decrease in the number of layers. In particular, when the applied load was increased beyond $300 \mathrm{MPa}$, the number of ordering layers was reduced from eight to seven for the $\mathrm{FeO}$, $\mathrm{Fe}_{2} \mathrm{O}_{3}(001)$, and $\mathrm{Fe}_{2} \mathrm{O}_{3}(012)$ surfaces (Figs. 3(b)-3(d)). The change in the number of liquid layers in the thin film, as a function of the film thickness, is in agreement with the previous work by Liu et al. [47]. 
One of the interfacial phenomena that has attacted considerable interest in thin-film lubrication investigations is the interfacial slip. This slip was determined as the velocity difference between the effective speed applied on the surface and an apparent one calculated from the lubricant layer at solidluid interfaces. The velocity profiles across the film thickness for each surface represented in Fig. 4 demonstrate that most of the velocity slip is observed for the $\mathrm{FeO}$ surface, whereas it is insignificant for $\mathrm{Fe}_{2} \mathrm{O}_{3}$ (001).

To quantify the slip phenomenon, a dimensionless parameter $s$ is measured based on the obtained apparent $\left(\dot{\gamma}_{\text {app }}\right)$ and effective $\left(\dot{\gamma}_{\text {eff }}\right)$ shear rates, as expressed in the following formula:

$$
s=1-\frac{\dot{\gamma}_{\text {eff }}}{\dot{\gamma}_{\text {app }}}
$$

The apparent shear rate is defined as $\dot{\gamma}_{\text {app }}=$ $\left(v_{1}-v_{2}\right) / h$ with $v_{i}$ as the sliding velocity of the surfaces and $h$ is the lubricant film thickness, whereas the effective shear rate is evaluated from the gradient of the velocity profile across the lubricant film thickness. The $s=0$ value indicates a non-slip with $\dot{\gamma}_{\text {eff }}=\dot{\gamma}_{\text {app }}$, while an ideal value of $s=1$ is for a full interfacial slip with $\dot{\gamma}_{\text {eff }}=0$. Figure 5 shows the linear relationship between this slip parameter and applied pressure. In addition, this slip also depends strongly on the crystalline structure in the order $\mathrm{Fe}_{2} \mathrm{O}_{3}(001)<\mathrm{Fe}(100)<\mathrm{Fe}_{2} \mathrm{O}_{3}(012)<\mathrm{FeO}$ (100). Fillot et al. [48] proposed that the slip varies linearly with the applied load. Therefore, a linear function was fit for the obtained MD results as expressed in the following equation:

$$
s=a \times p-b
$$

where $p$ is the applied pressure. The fitting results for the different surfaces presented in Fig. 5 show that the slope coefficients $a$ of $1.76,0.83,0.78$, and 0.26 , and constant parameters $b$ of $-0.11,0.55,0.30$, and 0.87 were obtained for $\mathrm{Fe}_{2} \mathrm{O}_{3}(001), \mathrm{Fe}_{2} \mathrm{O}_{3}(012)$, $\mathrm{Fe}$, and $\mathrm{FeO}$ surfaces, respectively. The results also
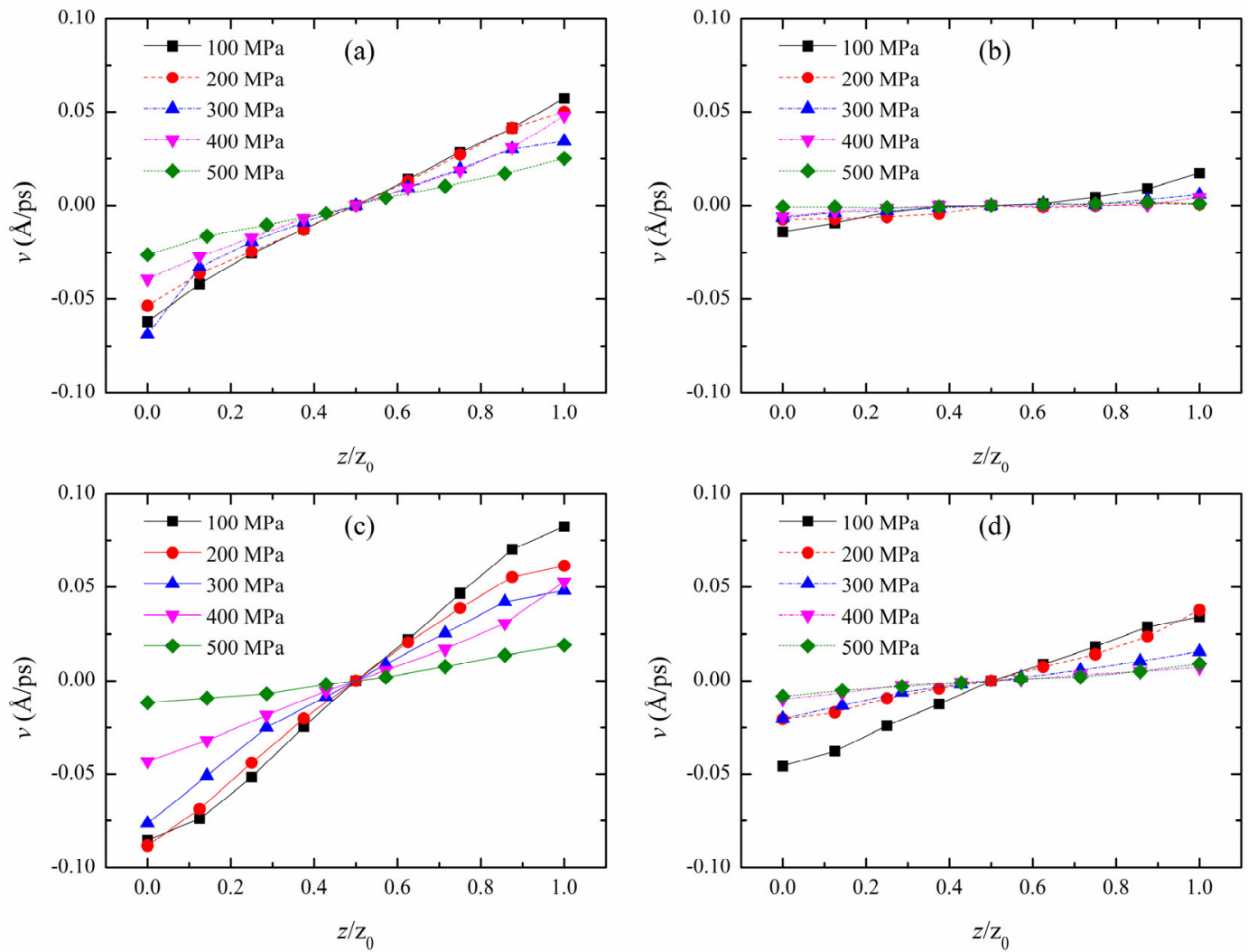

Fig. 4 Velocity profile across film thicknesses in the case of (a) Fe (100) surfaces, (b) $\mathrm{FeO}(100)$ surfaces, (c) $\mathrm{Fe}_{2} \mathrm{O}_{3}(001)$ surfaces, and (d) $\mathrm{Fe}_{2} \mathrm{O}_{3}$ (012) surfaces at different applied loads. 

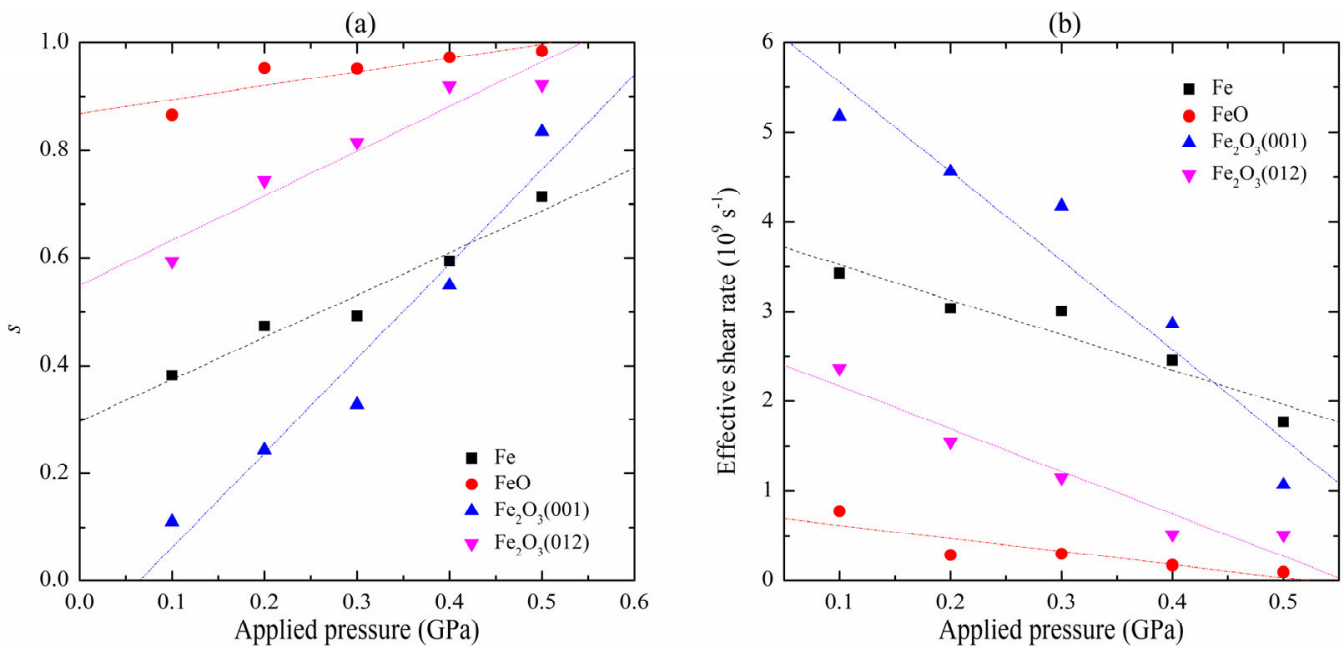

Fig. 5 Variation of (a) velocity slip $(s)$ and (b) effective shear rate $\left(\dot{\gamma}_{\text {eff }}\right)$ with respect to applied loads on different surface structures.

indicate that the onset of the slip could start at a small applied load for $\mathrm{FeO}, \mathrm{Fe}$, and $\mathrm{Fe}_{2} \mathrm{O}_{3}$ (012) surfaces, although this phenomenon only occurs when an applied load larger than $0.06 \mathrm{MPa}$ is imposed on the $\mathrm{Fe}_{2} \mathrm{O}_{3}$ (001) surface. This observation suggests that a practical implication to this phenomenon is that the thin film lubrication of hydrocarbon could be optimized by utilizing the smoother surfaces more than $\mathrm{Fe}_{2} \mathrm{O}_{3}$ (001), which is generally considered as the most stable facet of $\alpha-\mathrm{Fe}_{2} \mathrm{O}_{3}[49,50]$. It is also commonly found on steel surfaces of the high load and elevated temperature [51, 52]. Moreover, it also predicts that the full velocity slip at an applied load of $0.5 \mathrm{GPa}$ is for the FeO (100) surface, whereas the corresponding values of 0.54, 0.63, and $0.90 \mathrm{GPa}$ are for $\mathrm{Fe}_{2} \mathrm{O}_{3}(012), \mathrm{Fe}_{2} \mathrm{O}_{3}$ (001), and Fe (100), respectively. Because all tribosystems were sheared with similar effective shear rates $\dot{\gamma}_{\text {eff }}$, the slip parameter s could be considered as an inverse function of the apparent shear rate $\dot{\gamma}_{\text {app }}$. Indeed, Fig. 5(b) shows a contrasting tendency in that the effective shear rate decreases almost linearly with an increase in the applied load.

Friction is a critical component commonly considered in tribological investigations because it reflects the lubricity of the lubricant. In the current MD simulation, the shear stress was calculated as a fraction of frictional force, which was evaluated by the sum of the lateral forces on the surface exerted by the lubricant via the pairwise interfacial interactions over the surface area. This shear stress value was time-averaged during the last 5 ns for all cases, and the linear relationship between the calculated shear stress and an applied load is presented in Fig. 6. However, the figure shows a remarkable discrepancy in the CoF value for each surface material. Particularly, the respective $\mathrm{CoF}$ values of $0.009,0.055,0.027$, and 0.072 were found for $\mathrm{FeO}$, $\mathrm{Fe}_{2} \mathrm{O}_{3}(012)$, $\mathrm{Fe}$, and $\mathrm{Fe}_{2} \mathrm{O}_{3}$ (001) surfaces. Although the highest frictional force was observed on the $\mathrm{Fe}_{2} \mathrm{O}_{3}$ (001) surface, it was insignificant on the $\mathrm{FeO}$ surface. Surface corrugation played a vital role in the tribological performance of the hexadecane between

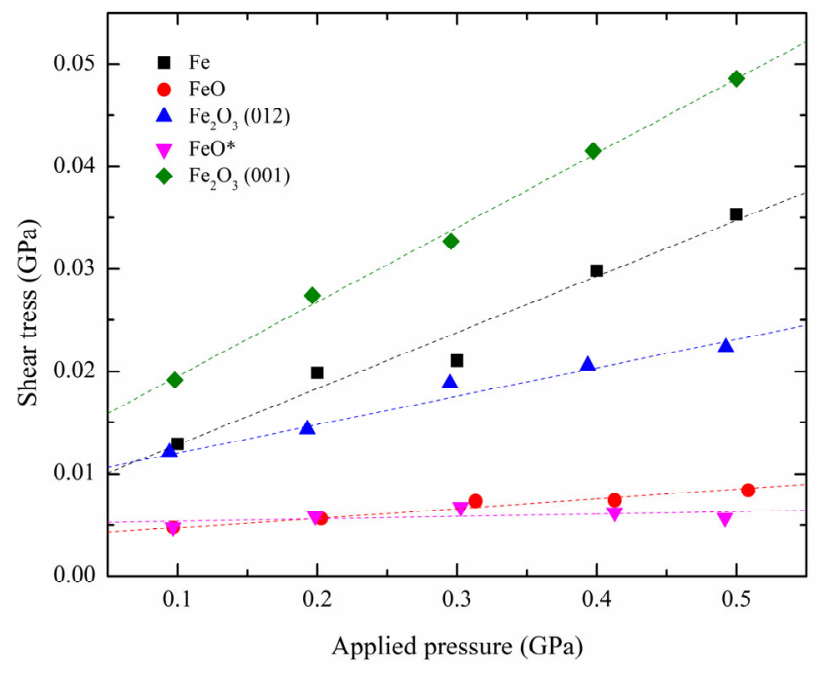

Fig. 6 Correlation between shear stress and applied load on different surface structures. 
$\mathrm{Fe}_{2} \mathrm{O}_{3}$ (001) surfaces. Indeed, the $\mathrm{FeO}, \mathrm{Fe}$, and $\mathrm{Fe}_{2} \mathrm{O}_{3}$ (012) surfaces were smoother, therefore, they could generate a lower $\mathrm{CoF}$ value than that of $\mathrm{Fe}_{2} \mathrm{O}_{3}(001)$. This result is in good agreement with that determined by Berro et al. [4] and Savio et al. [1].

Although $\mathrm{FeO}(100)$ also has a smooth surface similar to $\mathrm{Fe}$ (100), we observed that the CoF value of $\mathrm{FeO}(100)$ is lower than that of the pure iron surface. This is an interesting observation and an explanation for this phenomenon could be the commensurability of hexadecane with different surfaces. Thomson and Robbins [53] and Savio et al. [1] stated that a greater commensurability between a surface and fluid could lead to a greater enhancement of the interfacial slip. The current observation agrees with their studies because the gap between surface atoms on the $\mathrm{FeO}$ (100) surface was shorter than that on the Fe surface. In fact, gaps of 2.866 and $2.1865 \AA$ were determined for Fe (100) and FeO (100) surfaces, respectively.

Lubricant viscosity is a critical rheological property in thin-film lubrication. Under the confined gap, this physical component is different from the bulk phase owing to the ordering rearrangement of the lubricant molecules and the change in density. The effective viscosity $\eta_{\text {eff }}$ of hexadecane was calculated to observe the effect of the adhesive strength and surface properties on this rheological property. The obtained results are presented in Fig. 7, which shows

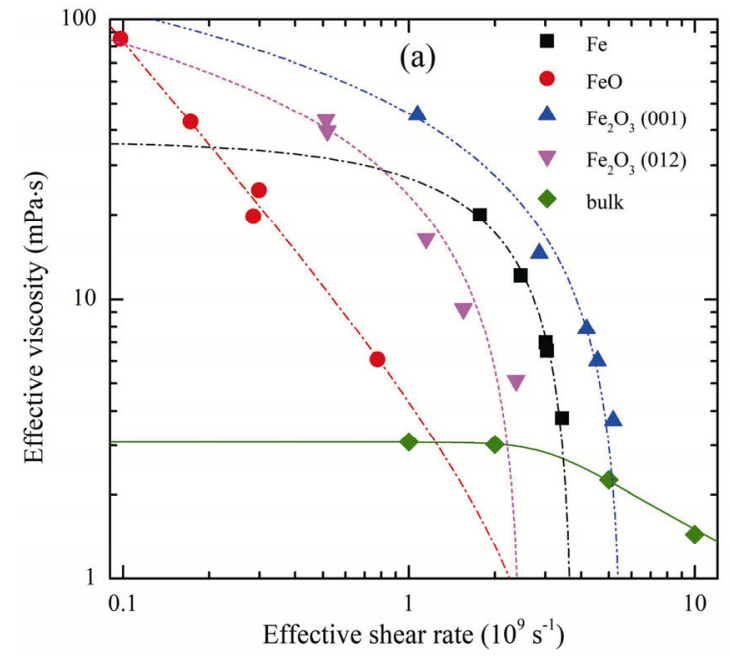

that the viscosity of hexadecane increases significantly with the applied load, especially in the case of $\mathrm{FeO}$ (100) and $\mathrm{Fe}_{2} \mathrm{O}_{3}$ (012) surfaces. Furthermore, this effective viscosity exhibits a large increase compared with the bulk value, thus implying that the hexadecane lubricant behaves more solid-like under extreme conditions. The results also show that the solid-like behavior is more pronounced on $\mathrm{FeO}$ and $\mathrm{Fe}_{2} \mathrm{O}_{3}$ (012) surfaces in comparison with $\mathrm{Fe}$ and $\mathrm{Fe}_{2} \mathrm{O}_{3}$ (001). The dependence of $\eta_{\text {eff }}$ on $\dot{\gamma}_{\text {eff }}$ or $p$ can be described as a power function in the following expression:

$$
\eta_{\mathrm{eff}}=\eta_{0}+a \times x^{b}
$$

where $x$ is the effective shear rate or applied load, $\eta_{0}$ is the effective viscosity at zero shear rate or zero-applied load, $a$ and $b$ are the parameters. The fitting results are plotted in Fig. 7.

\subsection{Influence of force fields}

The average density of $\mathrm{C}_{16} \mathrm{H}_{34}$ during the confined shear simulation was calculated, and the results were presented in Table 1 . The data reveal that classical FFs of OPLS UA and COMPASS result in higher densities than those obtained from the reactive FFs of AIREBO and REAXFF. COMPASS exhibits the best agreement with experimental values of $0.77 \mathrm{~g} / \mathrm{cc}$ [54], whereas other FFs show larger deviations up to $7 \%$.

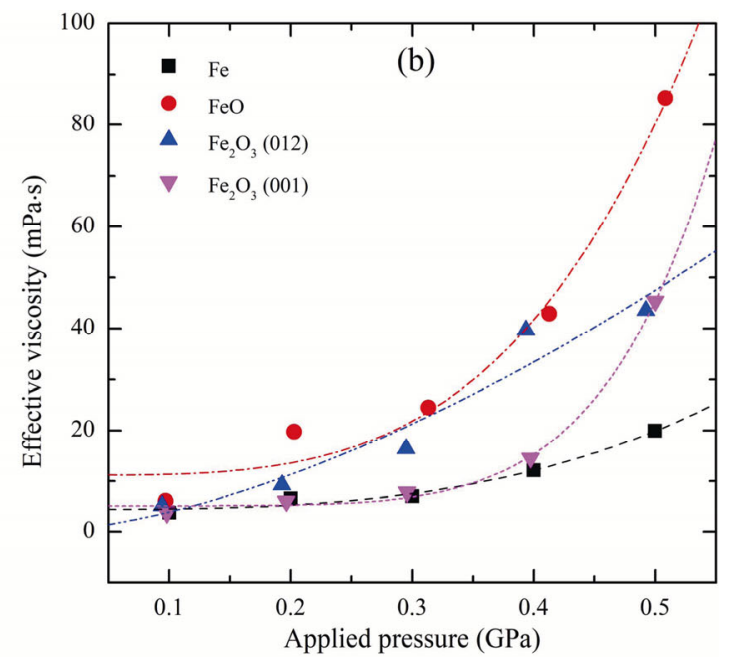

Fig. 7 Effective viscosity of hexadecane as a function of (a) effective shear rate and (b) applied load on different surface structures. 
Table 1 Comparison of time-average densities of $\mathrm{C}_{16} \mathrm{H}_{34}$ from different FFs.

\begin{tabular}{ccc}
\hline FFs & Density $(\mathrm{g} / \mathrm{cc})$ & Error $(\mathrm{g} / \mathrm{cc})$ \\
\hline OPLS-UA & 0.792 & 0.003 \\
COMPASS & 0.775 & 0.011 \\
AIREBO & 0.753 & 0.010 \\
ReaxFF & 0.715 & 0.004 \\
\hline
\end{tabular}

It should be noted that a larger number of surfaces have been used in this case to improve the reliability of our observations on the effect of used FFs on the hydrocarbon lubricating performance. The obtained results are presented in Figs. 8-10 for different surfaces, which indicate that the variation in densities across the film thickness for the UA model are much higher than those obtained from the AA models. Remarkably, in the middle regime of the lubricant, a significant decrease in density peaks is observed for AA models; in contrast, the decrease is insignificant for the UA model. These
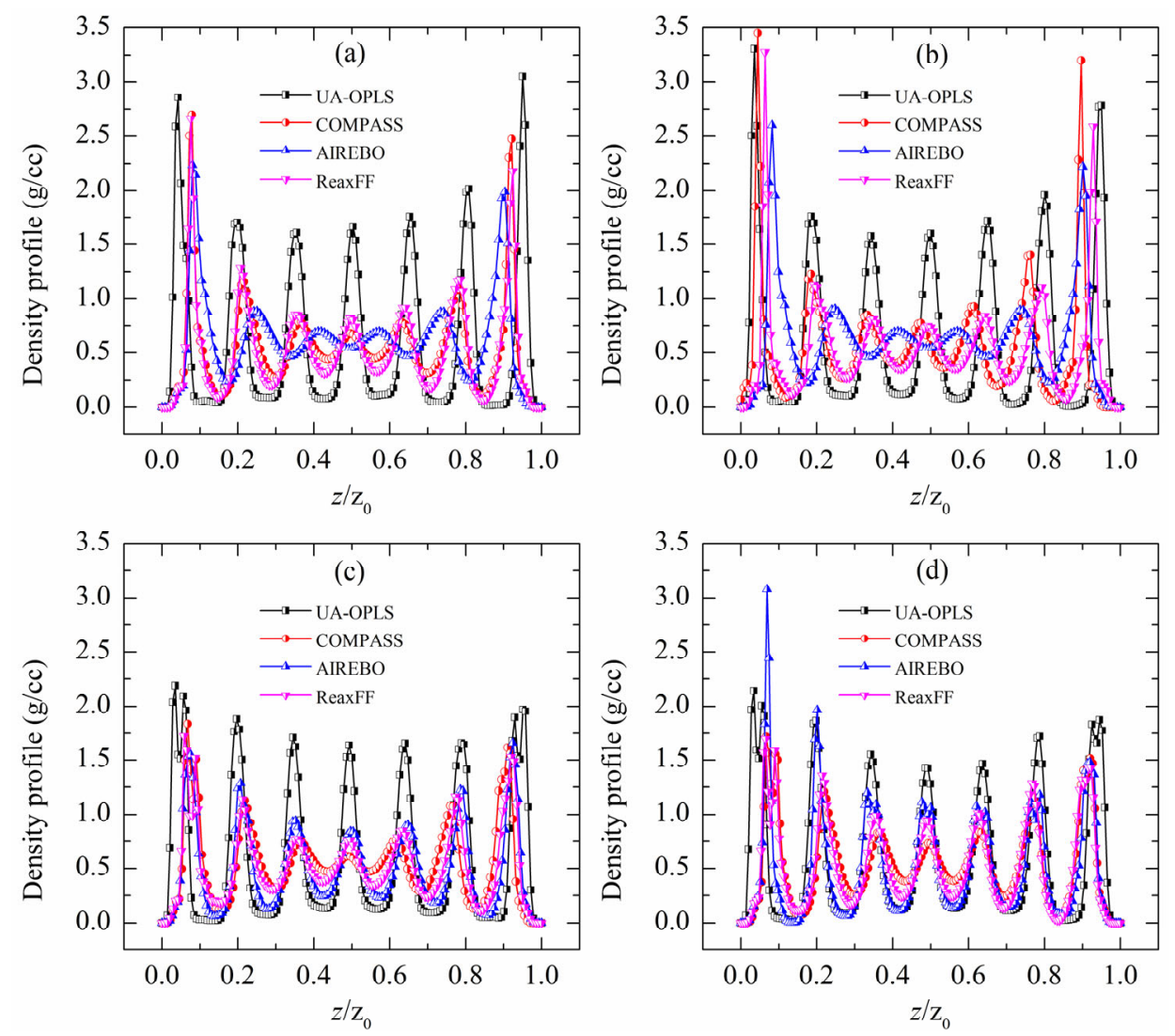

Fig. 8 Density profiles of $\mathrm{C}_{16} \mathrm{H}_{34}$ obtained from OPLS-UA, COMPASS, AIREBO, and ReaxFF on (a) $\mathrm{Fe}$ (100), (b) Fe (110), (c) Fe (111), and (d) Fe (111)(010) surfaces.

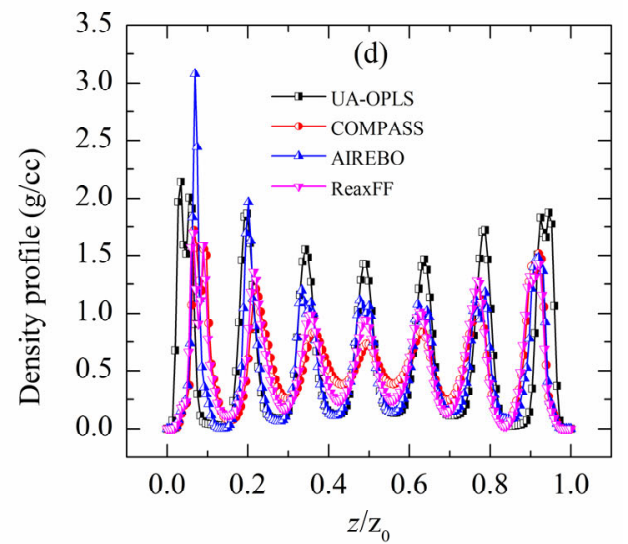

observations reveal in general, that the molecular structure of hydrocarbon or layering property, in particular, depends on a chosen FF.

The velocity profiles in Figs. 11-13 show distinct degrees of ordering for different FFs and surfaces. For instance, notable discrepancies were observed on Fe (100) (Fig. 11(a)), Fe (111)(010) (Fig. 11(d)), $\mathrm{FeO}(110)$ (Fig. 12(b)), and all $\mathrm{Fe}_{2} \mathrm{O}_{3}$ surfaces (Fig. 13). AIREBO FF showed the highest velocity gradient on Fe (100), whereas the velocity gradient for other FFs was insignificant. This observation is similar to that of $\mathrm{FeO}(110)$ in Fig. 12 and $\mathrm{Fe}_{2} \mathrm{O}_{3}$ (012) in Fig. 13. Besides the interfacial slip, which is found on smooth surfaces of Fe (110), Fe (111) sliding in the (100) direction, and $\mathrm{FeO}(100)$, the internal slip within hexadecane layers, is also found for reactive FFs of AIREBO and ReaxFF on the Fe (111)(010) surface as well as $\mathrm{Fe}_{2} \mathrm{O}_{3}$ (001). These results indicate that the FFs adopted to influence the tribological performance of the thin film. 

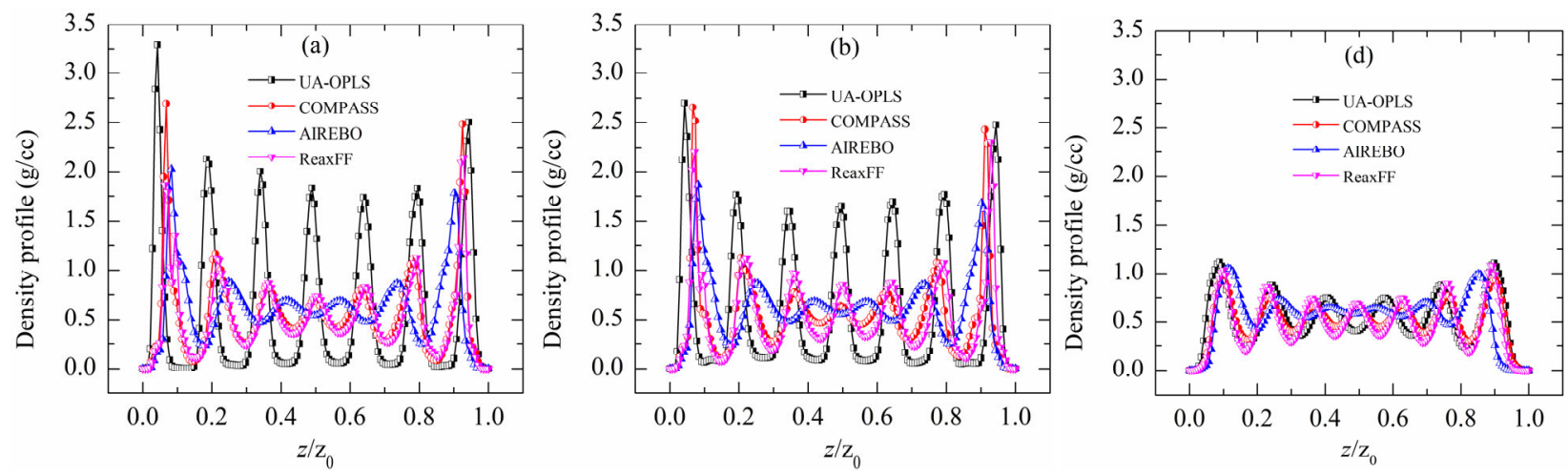

Fig. 9 Density profiles of $\mathrm{C}_{16} \mathrm{H}_{34}$ obtained from OPLS-UA, COMPASS, AIREBO, and ReaxFF on (a) FeO (100), (b) FeO (110), and (c) $\mathrm{FeO}(111)$ surfaces.
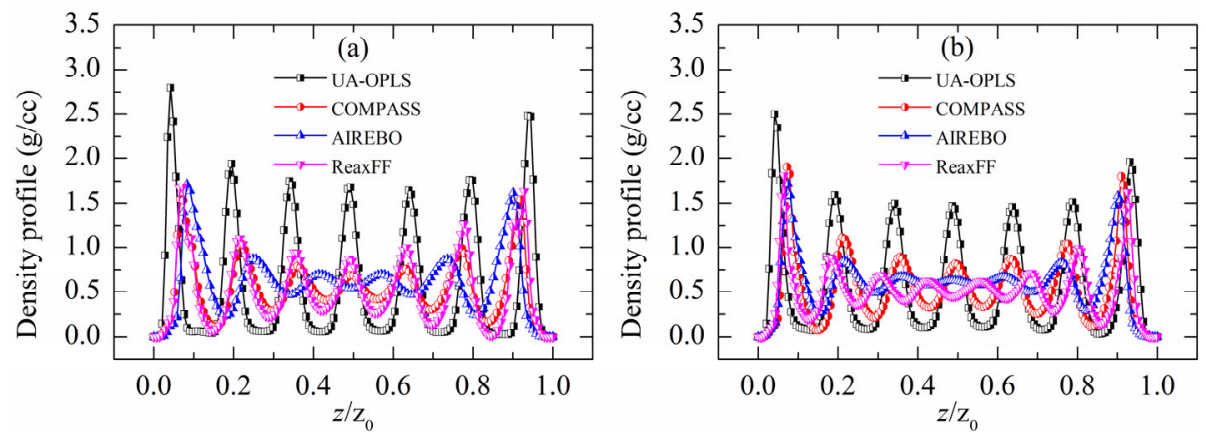

Fig. 10 Density profiles of $\mathrm{C}_{16} \mathrm{H}_{34}$ obtained from OPLS-UA, COMPASS, AIREBO, and ReaxFF on (a) $\mathrm{Fe}_{2} \mathrm{O}_{3}(001)$ and (b) $\mathrm{Fe}_{2} \mathrm{O}_{3}(012)$ surfaces.
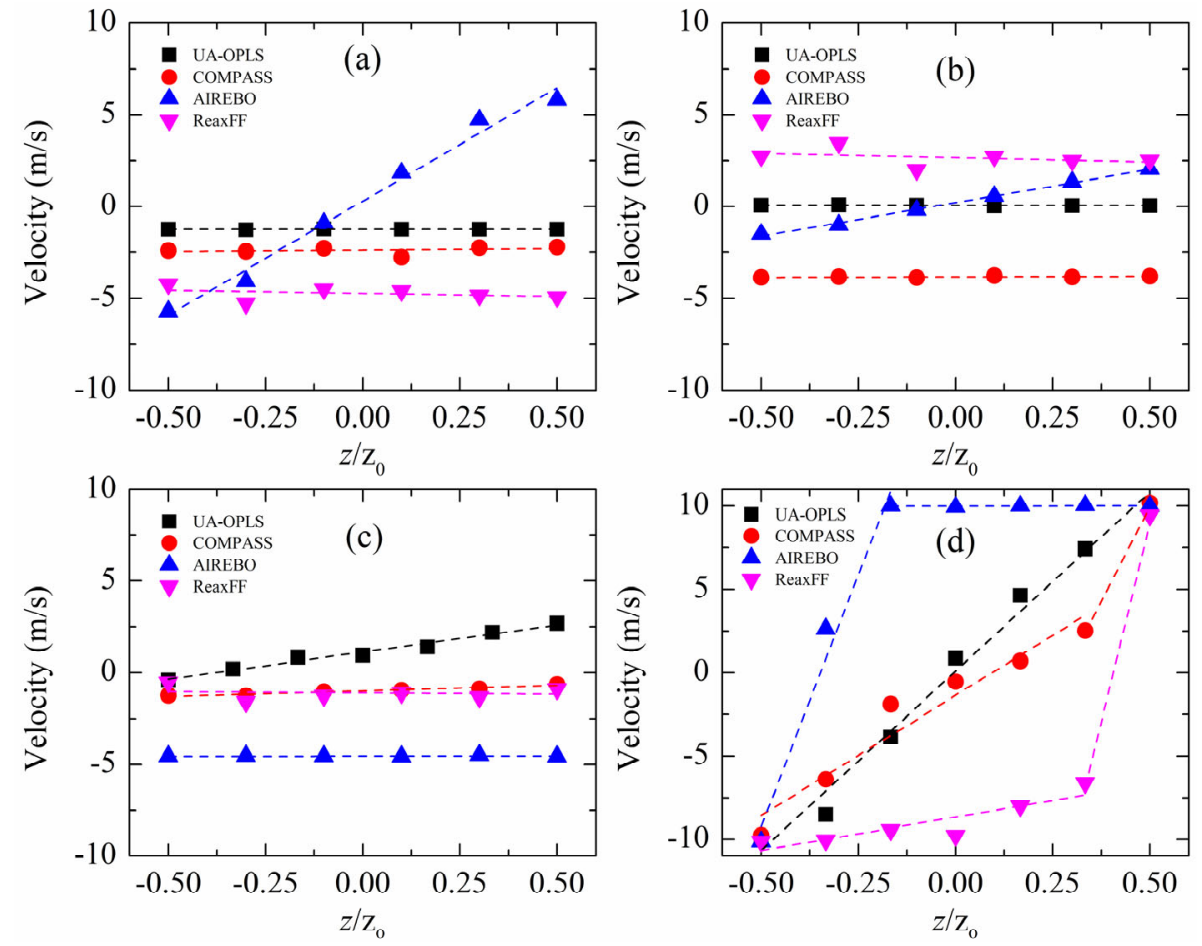

Fig. 11 Velocity profiles of $\mathrm{C}_{16} \mathrm{H}_{34}$ obtained from OPLS-UA, COMPASS, AIREBO, and ReaxFF on (a) $\mathrm{Fe}$ (100), (b) Fe (110), (c) Fe (111), and (d) Fe (111)(010) surfaces. 

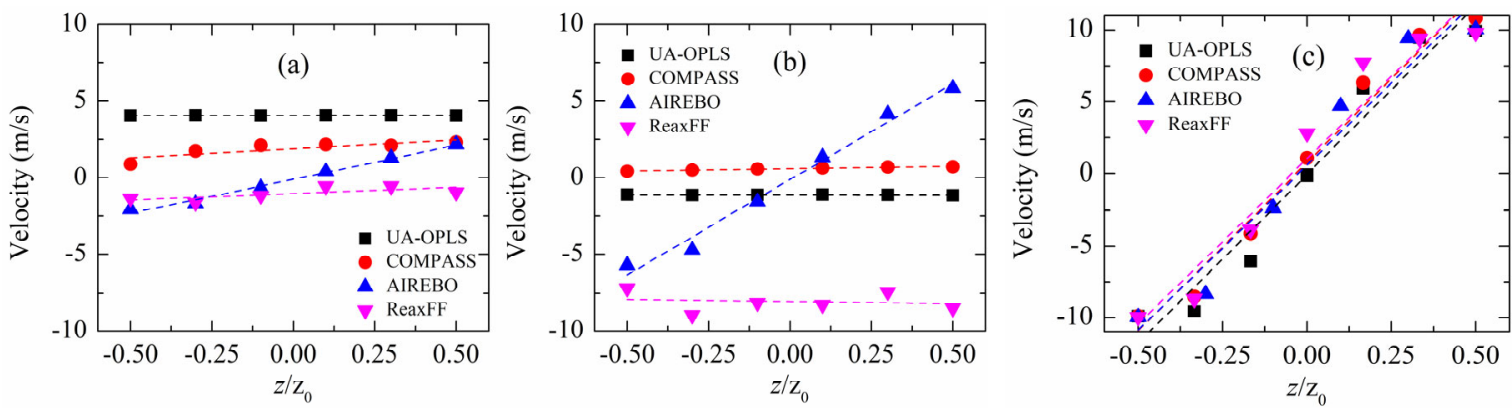

Fig. 12 Velocity profiles of $\mathrm{C}_{16} \mathrm{H}_{34}$ obtained from OPLS-UA, COMPASS, AIREBO, and ReaxFF on (a) FeO (100), (b) FeO (110), and (c) $\mathrm{FeO}$ (111) surfaces.
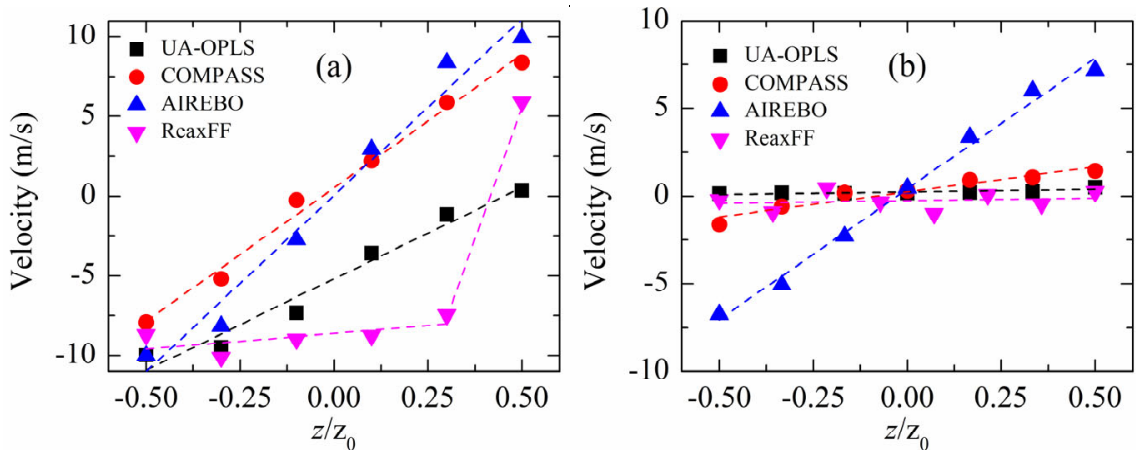

Fig. 13 Velocity profiles of $\mathrm{C}_{16} \mathrm{H}_{34}$ obtained from OPLS-UA, COMPASS, AIREBO, and ReaxFF on (a) $\mathrm{Fe}_{2} \mathrm{O}_{3}$ (001) and (b) $\mathrm{Fe}_{2} \mathrm{O}_{3}(012)$ surfaces.

In Ref. [22], we discovered that this slip phenomenon was mitigated by an increase in the surface potential parameter $(\zeta(\mathrm{kcal} / \mathrm{mol} / \AA))$, which is a general formula used to characterize the influence of surface properties, including surface energy, corrugation force, and commensurability height. Additionally, the $\zeta$ values increased in the order: $\mathrm{Fe}(110)<\mathrm{FeO}$ $(100)<\mathrm{Fe}(100)<\mathrm{Fe}_{2} \mathrm{O}_{3}(012)<\mathrm{Fe}(111)<\mathrm{FeO}(110)$ $<\mathrm{Fe}(111)(010)<\mathrm{Fe}_{2} \mathrm{O}_{3}(001)<\mathrm{FeO}$ (111), with respective values of $1.28,2.90,8.32,10.45,18.59$, $20.3,77.36,104.92$, and $154.4 \mathrm{kcal} / \mathrm{mol} / \AA$ [22]. To verify the transferability of our theory to different atomic models, the dependence of shear stress $\tau_{x z}$ $(\mathrm{MPa})$ and velocity slip ratio $s$ on $\zeta$ was analyzed, and the results are presented in Fig. 14. The results show that the current trends are consistent with previous observations in which $\tau_{x z}$ increases with $\zeta$, whereas $s$ decreases with an increase in $\zeta$.

However, the shear stresses vary significantly between different FFs, with the smallest values within $20 \mathrm{MPa}$ for AIREBO FF over various surfaces, whereas COMPASS FF exhibits results that are two to four times larger than AIREBO. Given the same surface properties and interfacial interactions between the surfaces and lubricants, the model of the lubricant could likely be the root cause of this difference. The less ordered structure, larger velocity gradient, and smaller shear stress reveal that AIREBO behaves more liquid-like than other FFs. In contrast, despite the OPLS-UA model producing smaller shear stresses than COMPASS and ReaxFF, the stronger layering structure (Figs. 8-10) and small velocity gradients (Figs. 11-13) verify that this kind of FF is more solid-like than the others. The error bar for ReaxFF is larger than that of other force fields owing to the larger deviation in the viscous friction within the lubricant.

With an increase in $\zeta$, the velocity slip when the lubricant is confined decreases. A similar concern has been aimed at shear stress, which is a critical property in lubrication. The determined values in Fig. 14(a) indicate an increase in shear stress with $\zeta$. Although the figure exhibits some fluctuation, the relationship presented is approximately linear. This observation is not only found in previous studies on COMPASS FF, but is also applicable to other atomic models in the current work. This indicates that our proposed $\zeta$ function can be considered as 

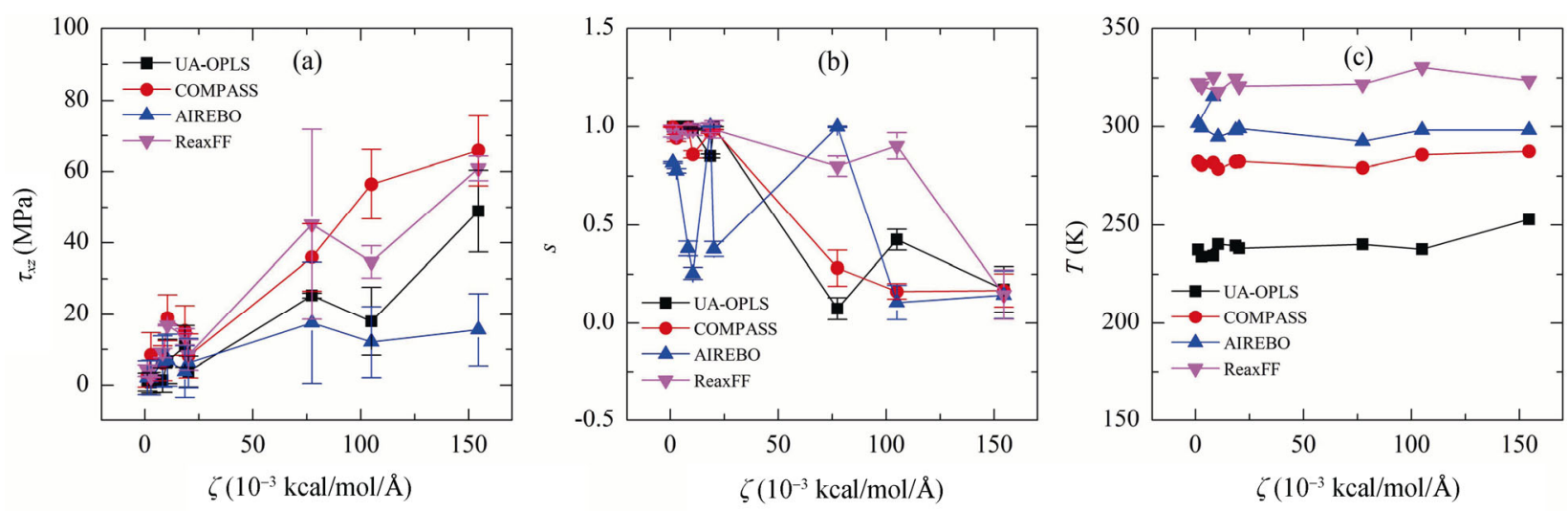

Fig. 14 Dependence of (a) shear stress, (b) velocity slip ratio and (c) lubricant temperature on characterized surface potential and employed FFs.

a general formula for characterizing the influence of surface properties, including surface energy, corrugation force, and commensurability height, on the tribological performance of lubricants at the atomic level.

The average lubricant temperature was also assessed and is presented in Fig. 14(c), which shows that the temperature generated within the lubricant by frictional heat is an independent function of the surface properties on smooth surfaces. As illustrated in Fig. 1, the thermostat with the NVT ensemble was only applied to the inner layer of the surface, such that temperature was induced from kinetic energy owing to sliding motion. The lowest temperature range of $230-250 \mathrm{~K}$ is determined for the OPLS-UA model. The higher $280-285 \mathrm{~K}$ values were evaluated for COMPASS FF. Reactive potentials result in larger temperature ranges of $295-320 \mathrm{~K}$ and $320-330 \mathrm{~K}$ for AIREBO and ReaxFF, respectively. The lower lubricant temperature observed for the OPLS-UA model is a direct consequence of the velocity slip at the interface because this slip reduces the kinetic energy transferred from the surface to the lubricant. The less transferred momentum from using the OPLS-UA model reduces the temperature of the lubricant to become smaller than other FFs. In essence, the atomic model provides a stronger layering structure and a smaller velocity gradient that will decrease frictional heat and lubricant temperature.

\section{Conclusions}

MD simulations were implemented to investigate the effects of applied load, chosen atomic models, and surface structure by adopting different crystalline structures of iron and iron oxides. The COMPASS force field with an AA model was used for hexadecane to study the influence of the applied load on the degree of ordering, shear stress, and velocity slip, which was addressed; whereas EAM and Buckingham potentials were applied to the surfaces. In addition, different potentials have been applied as lubricants to validate the transferability of our previously proposed surface potential parameter, which characterizes the influence of surface energy, corrugation force, and commensurability. The simulation results revealed as following:

1) The lubricant film became more solid-like with an increased applied load. In particular, there was an increase in the velocity slip, shear viscosity, and friction as the applied load increased. The degree of ordering structure also changes with the applied load but rather insignificantly. It is also remarkably dependent on the surface structure.

2) The chosen FFs significantly influence the performance of lubrication, rheological property, and molecular structure. With the smallest velocity slip, degree of ordering structure, and shear stress, AIREBO potential results in more liquid-like behavior compared with OPLS-UA, COMPASS, and ReaxFF. In general, classical potentials such as OPLS-UA and COMPASS behave more solid-like than reactive ones. Furthermore, the lubricant temperatures obtained from OPLS-UA and COMPASS are much lower than those of AIREBO and ReaxFF owing to their solid-like behavior.

3) The increase in shear stress and decrease in 
velocity slip with an increase in parameter $\zeta$ are still conserved for all chosen FFs, thus indicating that the proposed surface potential parameter $\zeta$ for COMPASS FF can be validated for a wide range of atomic models.

\section{Acknowledgements}

This project is supported by the Australian Research Council Discovery Projects DP170103173 and Linkage Project LP160101871. The authors would also like to thank the National Computational Merit Allocation Scheme (NCMAS) and Australia National Computational Infrastructure (NCI) for computing time on the High-Performance Computing Cluster through the UOW/NCI Partner Share Scheme.

Open Access This article is licensed under a Creative Commons Attribution 4.0 International Li-cense, which permits use, sharing, adaptation, distribution and reproduction in any medium or for-mat, as long as you give appropriate credit to the original author(s) and the source, provide a link to the Creative Commons licence, and indicate if changes were made.

The images or other third party material in this article are included in the article's Creative Commons licence, unless indicated otherwise in a credit line to the material. If material is not in-cluded in the article's Creative Commons licence and your intended use is not permitted by statutory regulation or exceeds the permitted use, you will need to obtain permission directly from the copyright holder.

To view a copy of this licence, visit http://creativecommons.org/licenses/by/4.0/.

\section{References}

[1] Savio D, Fillot N, Vergne P, Zaccheddu M. A model for wall slip prediction of confined n-alkanes: Effect of wall-fluid interaction versus fluid resistance. Tribol Lett 46(1): 11-22 (2012)

[2] Savio D, Fillot N, Vergne P. A molecular dynamics study of the transition from ultra-thin film lubrication toward local film breakdown. Tribol Lett 50(2): 207220 (2013)

[3] Zheng X, Zhu H T, Tieu A K, Kosasih B. Roughness and lubricant effect on 3D atomic asperity contact. Tribol Lett 53(1): 215-223 (2014)

[4] Berro H, Fillot N, Vergne P. Molecular dynamics simulation of surface energy and ZDDP effects on friction in nano-scale lubricated contacts. Tribol Int 43(10): 1811-1822 (2010)

[5] Berro H, Fillot N, Vergne P, Tokumasu T, Ohara T, Kikugawa G. Energy dissipation in non-isothermal molecular dynamics simulations of confined liquids under shear. J Chem Phys 135(13): 134708 (2011)

[6] Gao J P, Luedtke W D, Landman U. Structures, solvation forces and shear of molecular films in a rough nanoconfinement. Tribol Lett 9(1-2): 3-13 (2000)

[7] Jabbarzadeh A, Atkinson J D, Tanner R I. Effect of the wall roughness on slip and rheological properties of hexadecane in molecular dynamics simulation of couette shear flow between two sinusoidal walls. Phys Rev E 61(1): 690-699 (2000)

[8] Martini A, Vadakkepatt A. Compressibility of thin film lubricants characterized using atomistic simulation. Tribol Lett 38(1): 33-38 (2010)

[9] Wang C C, Chang R Y. Nonlinearity and slip behavior of n-hexadecane in large amplitude oscillatory shear flow via nonequilibrium molecular dynamic simulation. $J$ Chem Phys 136(10): 104904 (2012)

[10] Zheng X, Zhu H T, Kosasih B, Tieu A K. A molecular dynamics simulation of boundary lubrication: The effect of $n$-alkanes chain length and normal load. Wear 301(1-2): 62-69 (2013)

[11] Manias E, Subbotin A, Hadziioannou G, Ten Brinke G. Adsorption-desorption kinetics in nanoscopically confined oligomer films under shear. Mol Phys 85(5): 1017-1032 (1995)

[12] Cui S T, Cummings P T, Cochran H D. Molecular simulation of the transition from liquidlike to solidlike behavior in complex fluids confined to nanoscale gaps. J Chem Phys 114(16): 7189-7195 (2001)

[13] Manias E, Hadziioannou G, Bitsanis I, Ten Brinke G. Stick and slip behaviour of confined oligomer melts under shear. A molecular-dynamics study. EPL EurLett 24(2): 99-104 (1993)

[14] Stevens M J, Mondello M, Grest G S, Cui S T, Cochran $\mathrm{H}$ D, Cummings P T. Comparison of shear flow of hexadecane in a confined geometry and in bulk. $J$ Chem Phys 106(17): 7303-7314 (1996)

[15] Berro H. A molecular dynamics approach to nano-scale lubrication. Ph.D. thesis. Villeurbanne (France): INSA de Lyon, 2010.

[16] Hamza A V, Madix R J. The activation of alkanes on Ni(100). Surf Sci 179(1): 25-46 (1987)

[17] Dai Q, Gellman A J. A HREELS study of $\mathrm{C}_{1}-\mathrm{C}_{5}$ straight chain alcohols on clean and pre-oxidized $\operatorname{Ag}(110)$ 
surfaces. Surf Sci 257(1-3): 103-112 (1991)

[18] Wetterer S M, Lavrich D J, Cummings T, Bernasek S L, Scoles G. Energetics and kinetics of the physisorption of hydrocarbons on $\mathrm{Au}(111)$. J Phys Chem B 102(46): 9266-9275 (1998)

[19] Ta T D, Tieu A K, Zhu H T, Kosasih B. Adsorption of normal-alkanes on $\mathrm{Fe}(110), \mathrm{FeO}(110)$, and $\mathrm{Fe}_{2} \mathrm{O}_{3}(0001)$ : Influence of iron oxide surfaces. J Phys Chem C 119(23): 12999-13010 (2015)

[20] Sun H. COMPASS: an ab initio force-field optimized for condensed-phase applications-overview with details on alkane and benzene compounds. J Phys Chem B 102(38): 7338-7364 (1998)

[21] Cornell W D, Cieplak P, Bayly C I, Gould I R, Merz K M, Ferguson D M, Spellmeyer D C, Fox T, Caldwell J W, Kollman P A. A second generation force field for the simulation of proteins, nucleic acids, and organic molecules. J Am Chem Soc 117(19): 5179-5197 (1995)

[22] Ta D T, Tieu A K, Zhu H T, Kosasih B. Thin film lubrication of hexadecane confined by iron and iron oxide surfaces: A crucial role of surface structure. $J$ Chem Phys 143(16): 164702 (2015).

[23] Ewen J P, Gattinoni C, Thakkar F M, Morgan N, Spikes H A, Dini D. A comparison of classical force-fields for molecular dynamics simulations of lubricants. Materials 9(8): 651 (2016).

[24] Mendelev M I, Han S, Srolovitz D J, Ackland G J, Sun D Y, Asta M. Development of new interatomic potentials appropriate for crystalline and liquid iron. Philos Mag 83(35): 3977-3994 (2003)

[25] Plimpton S. Fast parallel algorithms for short-range molecular dynamics. J Comput Phys 117(1): 1-19 (1995)

[26] Guillot B, Sator N. A computer simulation study of natural silicate melts. Part I: Low pressure properties. Geochim Cosmochim Acta 71(5): 1249-1265 (2007)

[27] Guillot B, Sator N. A computer simulation study of natural silicate melts. Part II: High pressure properties. Geochim Cosmochim Acta 71(18): 4538-4556 (2007)

[28] Yeh C I, Berkowitz M L. Ewald summation for systems with slab geometry. $J$ Chem Phys 111(7): 3155-3162 (1999)

[29] Yue J, Jiang X C, Yu A B. Adsorption of the oh group on $\mathrm{SnO}_{2}(110)$ oxygen bridges: A molecular dynamics and density functional theory study. J Phys Chem C 117(19): 9962-9969 (2013)

[30] Prathab B, Subramanian V, Aminabhavi T M. Molecular dynamics simulations to investigate polymer-polymer and polymer-metal oxide interactions. Polymer 48(1): 409-416 (2007)

[31] Li C L, Choi P. Molecular dynamics study of the adsorption behavior of normal alkanes on a relaxed $\alpha-\mathrm{Al}_{2} \mathrm{O}_{3}$ (0001) surface. J Phys Chem C 111(4): 1747-
1753 (2007)

[32] Yue J, Jiang X C, Yu A B. Molecular dynamics study on $\mathrm{Au} / \mathrm{Fe}_{3} \mathrm{O}_{4}$ nanocomposites and their surface function toward amino acids. J Phys Chem B 115(40): 1169311699 (2011)

[33] Govender A, Curulla-Ferré D, Pérez-Jigato M, Niemantsverdriet H. First-principles elucidation of the surface chemistry of the $\mathrm{C}_{2} \mathrm{H}_{x}(x=0-6)$ adsorbate series on Fe(100). Molecules 18(4): 3806-3824 (2013)

[34] Zhao L F, Liu L C, Sun H. Semi-ionic model for metal oxides and their interfaces with organic molecules. $J$ Phys Chem C 111(28): 10610-10617 (2007)

[35] Berro H, Fillot N, Vergne P. Hybrid diffusion: An efficient method for kinetic temperature calculation in molecular dynamics simulations of confined lubricant films. Tribol Lett 37(1): 1-13 (2010)

[36] Jabbarzadeh A, Harrowell P, Tanner R I. Very low friction state of a dodecane film confined between mica surfaces. Phys Rev Lett 94(12): 126103 (2005)

[37] Wang X G, Weiss W, Shaikhutdinov S K, Ritter M, Petersen M, Wagner F, Schlögl R, Scheffler M. The hematite $\left(\alpha-\mathrm{Fe}_{2} \mathrm{O}_{3}\right)(0001)$ surface: Evidence for domains of distinct chemistry. Phys Rev Lett 81(5): 1038-1041 (1998)

[38] Trainor T P, Chaka A M, Eng P J, Newville M, Waychunas G A, Catalano J G, Brown Jr G E. Structure and reactivity of the hydrated hematite (0001) surface. Surf Sci 573(2): 204-224 (2004)

[39] Chambers S A, Yi S I. Fe termination for $\alpha-\mathrm{Fe}_{2} \mathrm{O}_{3}(0001)$ as grown by oxygen-plasma-assisted molecular beam epitaxy. Surf Sci 439(1-3): L785-L791 (1999)

[40] Jorgensen W L, Tirado-Rives J. The OPLS [optimized potentials for liquid simulations] potential functions for proteins, energy minimizations for crystals of cyclic peptides and crambin. J Am Chem Soc 110(6): 16571666 (1988)

[41] O'Connor T C, Andzelm J, Robbins M O. AIREBO-M: A reactive model for hydrocarbons at extreme pressures. J Chem Phys 142(2): 024903 (2015)

[42] van Duin A C T, Dasgupta S, Lorant F, Goddard W A. ReaxFF: A reactive force field for hydrocarbons. $J$ Phys Chem A 105(41): 9396-9409 (2001)

[43] Ohara T, Suzuki D. Intermolecular momentum transfer in a simple liquid and its contribution to shear viscosity. Microscale Thermophys Eng 5(2): 117-130 (2001)

[44] Ohara T, Torii D. Molecular dynamics study of thermal phenomena in an ultrathin liquid film sheared between solid surfaces: The influence of the crystal plane on energy and momentum transfer at solid-liquid interfaces. J Chem Phys 122(21): 214717 (2005)

[45] Kalyanasundaram V, Spearot D E, Malshe A P. Molecular dynamics simulation of nanoconfinement 
induced organization of $n$-decane. Langmuir 25(13): 7553-7560 (2009)

[46] Jabbarzadeh A, Harrowell P, Tanner R I. The structural origin of the complex rheology in thin dodecane films: Three routes to low friction. Tribol Int 40(10-12): 1574-1586 (2007)

[47] Liu X, Surblys D, Kawagoe Y, Bin Saleman A R, Matsubara H, Kikugawa G, Ohara T. A molecular dynamics study of thermal boundary resistance over solid interfaces with an extremely thin liquid film. Int $J$ Heat Mass Transfer 147: 118949 (2020)

[48] Fillot N, Berro H, Vergne P. From continuous to molecular scale in modelling elastohydrodynamic lubrication: Nanoscale surface slip effects on film thickness and friction. Tribol Lett 43(3): 257-266 (2011)

[49] Parkinson G S. Iron oxide surfaces. Surf Sci Rep 71(1):

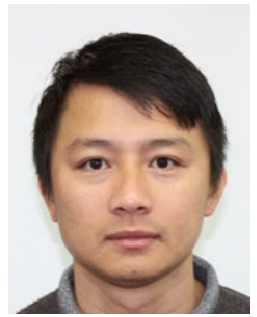

Thi D. TA. He currently works at the University of Wollongong as an associate research fellow. He received his honours bachelor degree in aeronautical engineering from Ho Chi Minh City University of Technology, Vietnam, in 2011.

He worked in PetroVietnam Technical Services

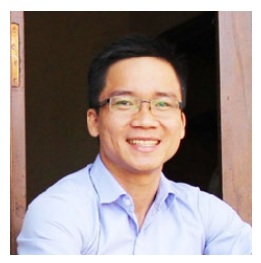

Hien D. TA. Currently, he works at Ho Chi Minh City University of Technology and Education, Vietnam. He received his bachelor, master, and Ph.D. degrees in physics from

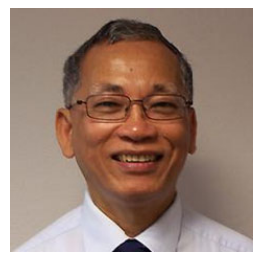

Kiet A. TIEU. He is currently a senior professor at the University of Wollongong. He is an academician of the Australian Academy of Technological Sciences and Engineering since 2007. He was awarded the William Johnson International Gold Medal in 2012 for lifetime achievements in materials processing research and teaching. He received Quangcheng
272-365 (2016)

[50] Kiejna A, Pabisiak T. Mixed termination of hematite $\left(\alpha-\mathrm{Fe}_{2} \mathrm{O}_{3}\right)(0001)$ surface. $J$ Phys Chem $C$ 117(46): 24339-24344 (2013)

[51] Chen R Y, Yuen W Y D. Oxide-scale structures formed on commercial hot-rolled steel strip and their formation mechanisms. Oxid Met 56(1-2): 89-118 (2001)

[52] Iordanova I, Surtchev M, Forcey K S, Krastev V. Hightemperature surface oxidation of low-carbon rimming steel. Surf Interface Anal 30(1): 158-160 (2000)

[53] Thompson P A, Robbins M O. Origin of stick-slip motion in boundary lubrication. Science 250(4982): $792-794$ (1990)

[54] Tanaka H, Yamaki Y, Kato M. Solubility of carbon dioxide in pentadecane, hexadecane, and pentadecane + hexadecane. J Chem Eng Data 38(3): 386-388 (1993)

Corporation Mechanical and Construction Joint Stock Company (PTSC M\&C), Vietnam, from 2011 to 2012. In 2017, he received his Ph.D. degree from School of Mechanical, Materials \& Mechatronic Engineering from University of Wollongong, Australia. His research areas cover quantum chemistry and molecular dynamics simulations of thin-film lubrication.

Volgograd State Technical University, Russia, in 2009, 2011, and 2014, respectively. His research areas cover quantum physics and molecular dynamics simulations of solids and 2D materials.

Award in 2011 by the Jinan Municipal government (China) for excellent collaborative research and the technology transfer with Jinan Steel and the Qilu Award by the Shandong provincial government (China) for outstanding international collaborative research in hot rolling technology. His research experience is in tribology, rolling technology, and computational mechanics. 
Bach H. TRAN. He received his honours bachelor degree in chemical engineering from Ho Chi Minh City University of Technology, Vietnam, in 2015. In 2019, he received his Ph.D. degree at the School of Mechanical, Materials
\& Mechatronic Engineering from University of Wollongong, Australia, where he currently works as an associate research fellow. His research areas cover tribology, rolling technology, and metal forming using advanced state-of-the-art characterisation techniques. 